\title{
Development and Validation of Structured COVID Perception Interview Guide (COPING) for Assessing the Acute Impact of COVID-19 Diagnosis
}

\author{
Roshan Sutar $^{1}$ Anuja Lahiri ${ }^{2}$ Gaurav Singh ${ }^{1} \quad$ Swanzil Chaudhary ${ }^{3}$ \\ ${ }^{1}$ Department of Psychiatry, All India Institute of Medical Sciences \\ (AIIMS), Bhopal, Madhya Pradesh, India \\ 2 Department of Community and Family Medicine, All India Institute \\ of Medical Sciences (AlIMS), Bhopal, Madhya Pradesh, India \\ ${ }^{3}$ Department of Radiodiagnosis, All India Institute of Medical \\ Sciences (AIIMS), Bhopal, Madhya Pradesh, India \\ Address for correspondence Roshan Sutar, MD, Department of \\ Psychiatry, All India Institute of Medical Sciences (AlIMS), Bhopal, \\ Madhya Pradesh, India (e-mail: roshidoc@yahoo.co.in). \\ J Neurosci Rural Pract 2022;13:196-203.
}

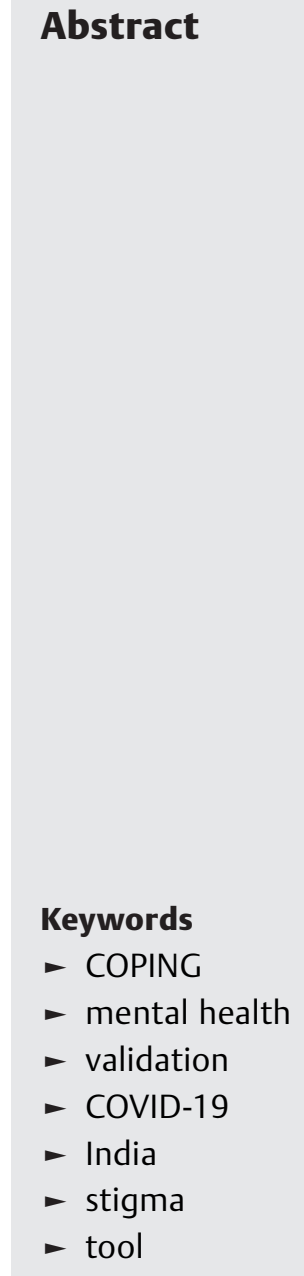

published online February 18, 2022
Objective Structured COVID Perception INterview Guide (COPING) is a novel tool developed to understand the acute impact after receiving the diagnosis of coronavirus disease 2019 (COVID-19) in the Indian setting. The approach carries importance for interviewing patients in a state of shock immediately after receiving the diagnosis of COVID. The tool is developed emphasizing the grief, stigma, and acute psychological perception in the immediate aftermath of receiving the positive test results of COVID-19. Since COVID-19 is characteristically different from other infectious illnesses, a structured interview guide could help to address the concerns related to acute loss of health.

Materials and Methods This study follows a mixed method design conducted from August 2020 to January 2021. In-depth telephonic interviews with mild to moderate COVID patients admitted to a tertiary hospital in central India was followed by development of COPING questionnaire.

Statistical Analysis Item-Content Validity Index (I-CVI) and Scale-Content Validity Index Universal Agreement (S-CVI/UA) was computed. Factor analysis, Bartlett's test, and Kaiser-Meier-Olkin measure of sampling adequacy was performed. Principal component analysis, scree plots, and parallel analysis with varimax rotation was used to determine the number of factors to extract. For measuring internal consistency, Cronbach's $\alpha$ was computed.

Results Out of 40 items, the final tool had 15 items after computing content validity, performing factor analysis and achieving desired level of internal consistency (Cronbach's $\alpha=0.702$ ). Five domains identified after factor analysis were awareness, grief/bereavement, stigma, social reciprocity, and stress adaptation/coping.

Conclusion COPING is a valid and reliable interview guide for Indian setting that will allow the assessment of perception of patients with acute COVID-19 infection. Taking into consideration the mental health implications of COVID-19, the availability of such a validated and reliable tool is a timely step to address the public health problem and assist the ongoing research on COVID-19 and similar illnesses in the future.
DOI https://doi.org/

10.1055/s-0041-1742232. ISSN 0976-3147.

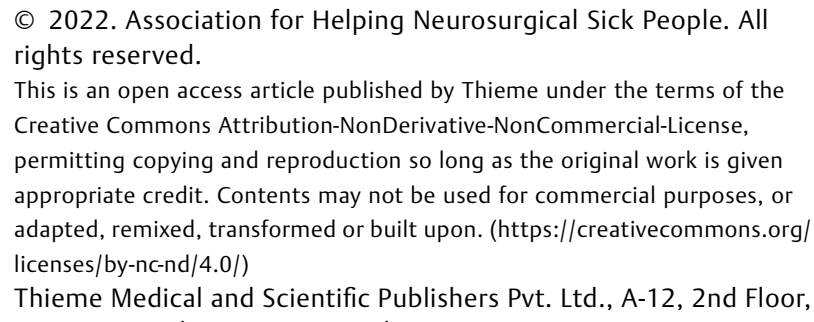

(c) 2022. Association for Helping Neurosurgical Sick People. All rights reserved.

This is an open access article published by Thieme under the terms of the Creative Commons Attribution-NonDerivative-NonCommercial-License, permitting copying and reproduction so long as the original work is given appropriate credit. Contents may not be used for commercial purposes, or adapted, remixed, transformed or built upon. (https://creativecommons.org/ licenses/by-nc-nd/4.0/)

Thieme Medical and Scientific Publishers Pvt. Ltd., A-12, 2nd Floor, Sector 2, Noida-201301 UP, India 


\section{Introduction}

The entire world has seen the rapid spread of pandemic due to novel coronavirus -19 over the year and half. ${ }^{1-6}$ As per the World Health Organization, in India, between January 3, 2020 and August 12, 2021 there were 32,036,511 confirmed cases of coronavirus disease-19 (COVID-19) with 429,179 deaths. ${ }^{7,8}$ Infection rates and acute psychological effects after testing positive for COVID-19 are significant despite various measures taken in India to contain the spread of the virus including stepwise lockdown, social distancing, and strict personal hygiene. ${ }^{9-11}$ The immediate state of shock resulting from the diagnosis of COVID-19 is multidimensional and involves acute loss of health (grief), anticipation about course of illness, doubts regarding results of the test, ruminations about close contacts, the perceived guilt of social victimization, fear of quarantine/isolation, the stigma of illness, and the anxiety of losing occupation. ${ }^{12-19}$ The evidence supporting the effective control of the pandemic rests on individual responsible behavior that in turn is affected by the person's mental status immediately after testing positive for COVID-19. ${ }^{20-22}$ The process of bereavement depends on the resilience of an individual including psychological acceptance of the test results and coping mechanism toward accepting the treatment protocol. $^{23-26}$ The disturbed mental status of an individual is further exaggerated if the concerns are not addressed through reliable information instantly along with handling and acknowledging the emotional turmoil during the spread of COVID-19 infection before the test results are declared. ${ }^{27,28}$ What happens to the mental status of people immediately after testing positive for COVID-19 is an unexplored area so far and requires systematic exploration. The consultation-liaison psychiatry teams at general hospitals have been working with mental health concerns of patients with COVID-19 and are trying to identify issues beyond depression and anxiety disorders. The exploration of the mental status of such individuals requires a gentle approach with a structured interview to address the unawareness, guilt, stigma, psychological trauma, coping, and grief associated with COVID-19.

Understanding the perception of positive test results of COVID-19 is an important but underrecognized area of research. Currently, there are no interview guides available that can help us to understand their trajectory of mental health. It is, therefore, paramount to understand the acute mental status of such individuals and attempt to make an interview guide in the Indian setting.

\section{Materials and Methods}

A mixed-method design was adopted. The initial set of items was generated through in-depth interviews and the items generated were tested through content validity. The final structured tool was factor analyzed which comprised the quantitative aspect.

\section{Qualitative Aspect}

The research protocol was approved by the institutional ethics committee (IM0276). The data collection process was done between August 2020 and January 2021. A list of subjects with contact details, meeting the inclusion criteria was collected from the acute infection ward by one of the investigators. We followed the Standards for Reporting Qualitative Research for reporting the findings of the in-depth interviews.

\section{Qualitative Approach and Research Paradigm}

Grounded theory was used for the initial 10 in-depth telephonic interviews ( - Supplementary Table S1 [available in the online version only], - Fig. 1) along with a thorough literature search for the generation of the initial 40 items in the tool (-Supplementary Table S2, available in the online version only). Grounded theory befittingly assisted in the creation of items based on the discussions with the recently diagnosed COVID-19 patients. Grounded theory with symbolic interactionism research paradigm was used as it is one of the finest ways to elucidate the interaction of actions to settings.

\section{Researcher Characteristics and Reflexivity}

Research team members who conducted in-depth interviews involved one psychiatrist and three nonpsychiatrist researchers trained for neutral interview techniques. Interviewing and data collection were done by nonpsychiatrist researchers. This ensured that their profession, experiences, or prior assumptions do not influence the interactions with participants. All the telephonic interviews were scheduled keeping in view the convenience of the participants and researchers. The interview varied from 35 to 70 minutes.

\section{Interview Site and Setting}

The in-depth interviews were conducted telephonically with participants admitted in a hospital setting considering the safety protocols and social distancing norms.

\section{Sampling Strategy}

We conducted 10 in-depth interviews. Due to thematic saturation, no further sampling was required. Few narratives are described in -Supplementary Fig. S1, available in the online version only.

\section{Data Collection Methods, Instrument, and Processing} The interviews were conducted using interview guides (-Supplementary Table S1, available in the online version only) and were recorded with help of smartphone recorders. All the verbatims were transcribed in Hindi language. The confidentiality of the participants was maintained by allocating Unique Identification Numbers to each participant. Three separate researchers read and reread the transcripts to generate themes and codes to be incorporated as items in the tool. This was further triangulated with an exclusive literature review.

\section{Unit of Study}

All COVID-19 patients (reverse transcription polymerase chain reaction confirmed in the past 48 hours) with asymptomatic/mild and moderate to severe nature of the disease, who were aged above 18 years were included in the study. We excluded patients who required oxygen therapy/ intensive care/inotropes and/or were pregnant. 


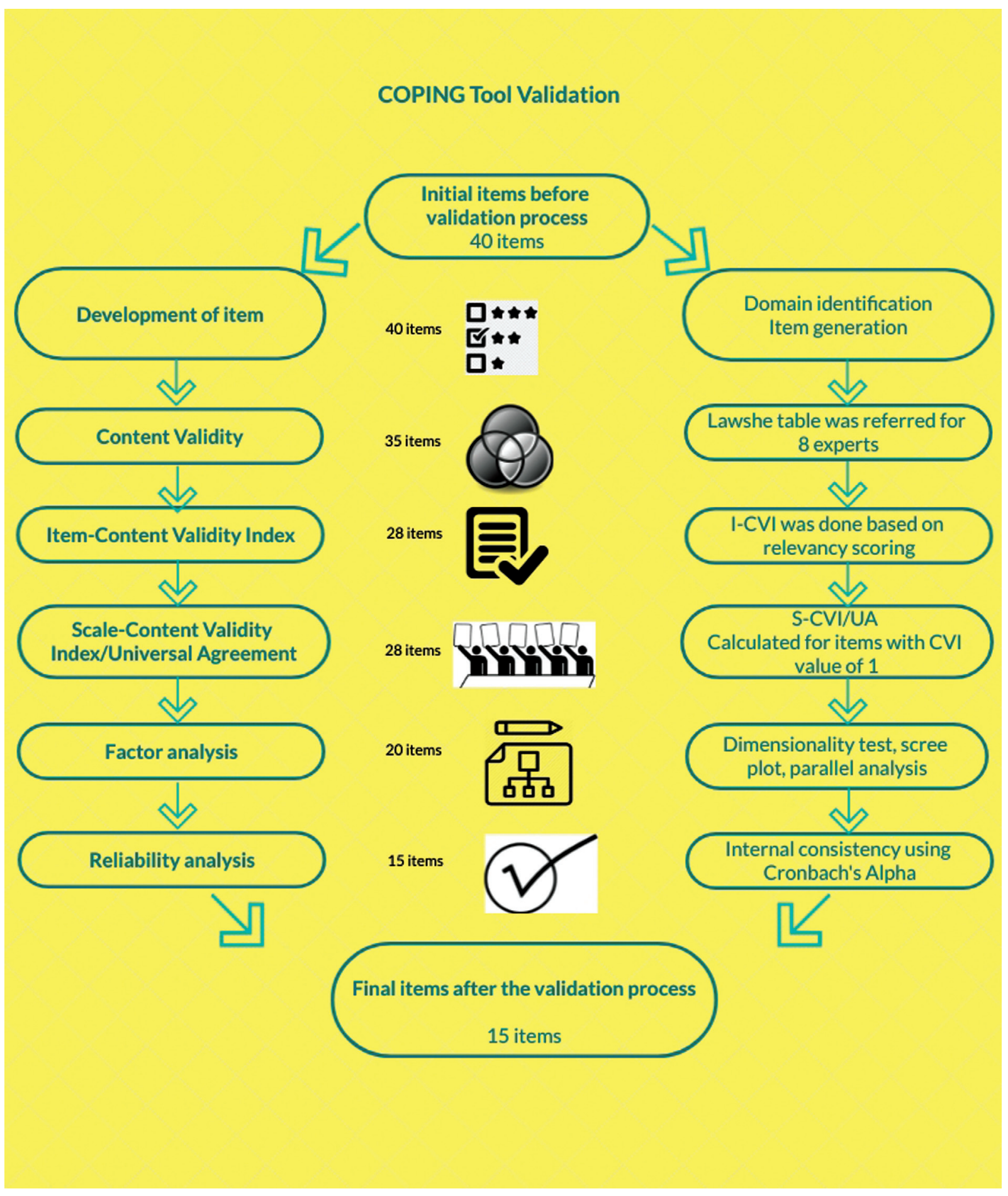

Fig. 1 Steps conducted for tool validation.

\section{Quantitative Aspect}

The responses to the items were on a 5-point Likert scale ( $1=$ strongly disagree to $5=$ strongly agree). Content Validity Ratio (CVR), Item-Content Validity Index (I-CVI), and ScaleContent Validity Index/Universal Agreement (S-CVI/UA) were calculated from the responses of eight subject experts (psychiatrists and physicians). After this process, the 40 -item tool was reduced to 28 items (based on scoring in - Supplementary Table 52, available in the online version only) which were applied online through emails and WhatsApp numbers using an online link through the KoBo Toolbox. An online informed consent was obtained by three researchers from the department of psychiatry. The team monitored and supervised the data collection process and maintained a separate log of patients who denied consent. Participants who were denied initially were given an opportunity for the second time.

KoBo Toolbox permits to availability of the features publicly or with limited users based on the choice of the researcher (GNU licensed). This is an open-source, smartphone-based data collection tool that can also be operated on a desktop. The 
researcher/organization can use the services by creating a free account (https://www.kobotoolbox.org/privacy/).

Factor analysis, reliability analysis, and construct validity were computed from the responses of 112 study participants which reduced the tool further to 15 items divided into 5 broad categories, namely, awareness, grief/bereavement, stigma, social reciprocity, and stress adaptation/coping.

Step 1: Identification of a domain

It was done to enable the process of item generation by a specification of domain boundaries. Domains were identified after 10 in-depth interviews of prospective scale users (inductive method) and literature review (deductive method).

\section{Step 2: Calculating CVR}

After identification of domain and generation of items, CVR was estimated by opinion from 8 subject experts (-Supplementary Table S3, available in the online version only) using the formula CVR $=(\mathrm{Ne}-\mathrm{N} / 2) /(\mathrm{N} / 2)$ where an item with a value above 0.75 was considered. Here, Ne was the number of experts indicating an item as essential and $\mathrm{N}$ was the total number of experts. Lawshe Table was referred for a cutoff value of 0.75 for 8 panelists (-Supplementary Table S4, available in the online version only).

\section{Step 3: Calculation of I-CVI}

After the computation of CVR, I-CVI was calculated using the formula I-CVI $=$ (No. of experts giving score 3 or 4 for relevancy)/ Total no. of experts. Items with I-CVI more than $79 \%$ were considered as "Appropriate items," between 70 and 79\% were items in which minor revisions were required, and items with ICVI less than 70\% were eliminated (- Supplementary Table S5, available in the online version only).

\section{Step 4: Computation of S-CVI/UA}

Following this, S-CVI/UA was calculated using the formula $\mathrm{S}-\mathrm{CVI} / \mathrm{UA}=$ No. of items with $\mathrm{CVI}=1 /$ Total no. of items.

Step 5: Factor analysis

Factor analysis was conducted for the 28 items finalized based on CVI. Here, the 112 scale users rated each of the 28 items on a 5 -point Likert scale ( $1=$ strongly disagree, $2=$ disagree, 3 = don't know, 4 =agree, $5=$ strongly agree). After this Bartlett's test, Kaiser-Meier-Olkin measure of sampling adequacy (KMO MSA) was conducted. This was followed by the scree test and parallel analysis. Lastly, the factors were described based on the varimax rotation.

Step 5: Reliability analysis

Following this, internal consistency was measured using Cronbach's $\alpha$ statistics. This calculated the degree to which the responses are consistent across the items in each of the categories of factors. Reliability was computed with consideration of item deletion.

\section{Statistical Analysis and Results}

A total of 291 patients were contacted and 112 consented to participate in the research. The reasons for the exclusion of 179 subjects were unavailability of cellular networks, deterioration of illness, and inability to use smartphones (-Fig. 2).

The sociodemographic details of the 112 study participants are described in -Table 1.

Out of 112 , the majority of the study participants was male (70.5\%) and belonged from urban areas (84.8\%). About $48.2 \%$ were aged between 26 and 50 years. Around 33\% were in service (either government or private).

Based on in-depth interviews and literature review 40 items were generated of which 5 had CVR below 0.75 and hence were discarded (- Supplementary Table S3, available in the online version only). Out of 35 remaining items, 7 were eliminated as they had I-CVI less than 70\% (- Supplementary Table 55, available in the online version only). Thus, the total items were reduced to 28 after this step. Twenty-three of the 28 items had I-CVI equal to $100 \%$ or a value of one. The remaining five items were in the range of "Appropriate" which were revised after consultation and changes suggested by the experts. After calculation of I-CVI, S-CVI/UA was calculated which was $82.1 \%$. All the items were scored as "strongly disagree $=1$, disagree $=2$, neutral $/$ undecided $=3$, agree $=4$, and strongly agree $=5$." Further to maintain the robustness of the tool we reverse coded the items with positive connotation using the "transform into same variable command" in Statistical Package for the Social Sciences version 17 (SPSS-17). Factor analysis was conducted as Bartlett's test was found to be significant $(<0.001)$ depicting that it is suitable for the matrix to factor analyze and inferring that the correlation matrix differed significantly from the identity matrix. KMO MSA (for factoring above the value of 0.50 ) was performed. The KMO MSA value was above 0.50 (equal to 0.664 ) indicating the matrix is suitable for factoring (-Table 2 ).

Scree plot suggested that close to factors 4 and 5, the data can be summarized as there is evident leveling after factor 5 (-Fig. 3). Based on the parallel analysis we used a 5-factor solution which was categorized using varimax rotation. Based on factor analysis, a total of eight items were removed (item number 1, 2, 4, 17, 20, 22, 23, 26). Now 28 items were reduced to 20 items (- Supplementary Table 56, available in the online version only). The desired level of consistency (Cronbach's $\alpha=0.702$ ) was obtained by deletion of items $14,21,6,5$, and 7 (-Supplementary Table 57, available in the online version only). Thus, the final tool had 15 items (-Supplementary Table s8, available in the online version only).

\section{Description of Five Broad Categories Based on Factor Analysis}

1. Awareness: An individual's ability to recognize the status of COVID-19 infection, risk of transmission, recommended safety measures, and knowledge about the availability of the treatment.

2. Grief/Bereavement: The process of emotional expression of an infected individual over time, ranging from a state of shock to acceptance of the current health status.

3. Stigma: The level of marked discrimination associated with the infection, current health status, and internalization of societal responses. 


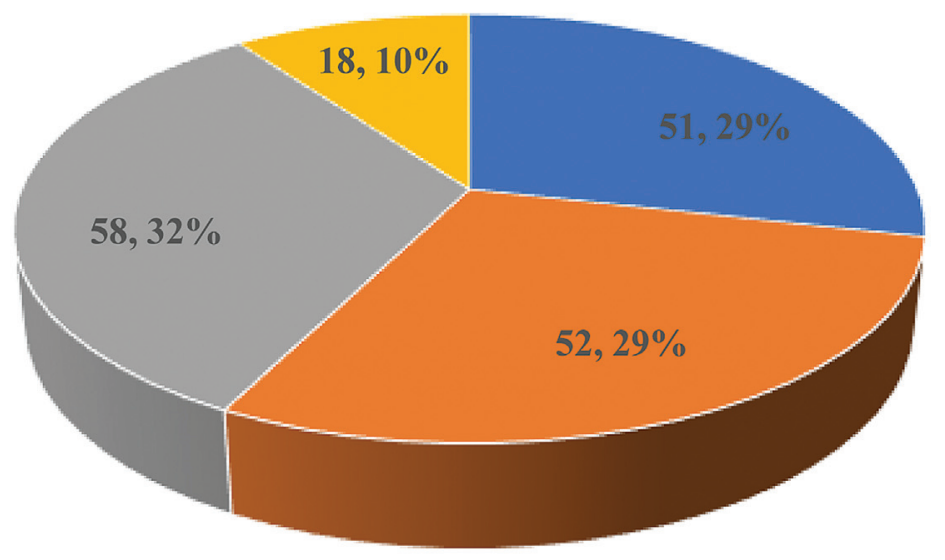

- Stable for the consent but later deteriorated and required oxygen/ICU admission hence could not interview

- Unavailability of the cellular Network in certain wards

- Not responded despite repeated calls

- Denied because 'Subjects not being user friendly with mobile/WhatsApp/Mail'

Fig. 2 Log of patients who could not be included in the study.

Table 1 Sociodemographic details of study participants

\begin{tabular}{|l|l|l|l|}
\hline Category & Subcategory & Number & Percentage \\
\hline \multirow{4}{*}{ Gender } & Male & 79 & 70.5 \\
\cline { 2 - 4 } & Female & 33 & 29.5 \\
\hline \multirow{5}{*}{ Area } & 25 or less & 25 & 22.3 \\
\cline { 2 - 4 } & $26-50$ & 54 & 48.2 \\
\cline { 2 - 4 } & 51 or above & 33 & 29.5 \\
\hline \multirow{5}{*}{ Occupation } & Urban & 95 & 84.8 \\
\cline { 2 - 4 } & Rural & 17 & 15.2 \\
\cline { 2 - 4 } & Student & 14 & 12.5 \\
\cline { 2 - 4 } & Service & 37 & 33 \\
\cline { 2 - 4 } & Laborer & 12 & 10.7 \\
\cline { 2 - 4 } & Homemaker & 18 & 16.1 \\
\cline { 2 - 4 } & Other & 31 & 27.7 \\
\hline
\end{tabular}

Table 2 KMO and Bartlett's test

\begin{tabular}{|l|l|}
\hline $\begin{array}{l}\text { Kaiser-Meyer-Olkin measure of } \\
\text { sampling adequacy }\end{array}$ & 0.664 \\
\hline Approx. chi-square & 968.334 \\
\hline Bartlett's test of sphericity (df) & 378 \\
\hline Significance & 0.000 \\
\hline
\end{tabular}

Abbreviations: df, degree of freedom; KMO, Kaiser-Meyer-Olkin.
4. Social reciprocity: An ability of an infected individual to have a healthy and balanced interaction with his family members, relatives, peers, and colleagues concerning psychosocial responsibilities.

5. Stress adaptation/coping: The patterns of psychological responses under stressful conditions leading to anxiety, excessive worrying, excessive spirituality, and additional personalized coping mechanisms.

Final tool (translated in the English language): -Supplementary Table S9, available in the online version only.

\section{Discussion}

The present tool is a validated and reliable interview guide for interviewing and measuring the acute impact of COVID-19 infection in the Indian setting. Though a plethora of studies and reviews have examined the broader mental health aspects of the general population, health care workers, and COVID-19 patients, ${ }^{29-32}$ less attention has been paid toward the development of a tool to understand the acute impact of COVID-19 diagnosis in the Indian context. The studies done so far have focused on symptoms of depression, anxiety, stress, and trauma and thus failed to capture the subtle psychological domains that were distinctly highlighted in qualitative interviews such as awareness, grief/bereavement, stigma, social reciprocity, and stress adaptation/coping. ${ }^{33-36}$ It is also practically appropriate to not include the clinical diagnosis of newonset depressive or anxiety disorders within 48 hours of diagnosis of COVID-19 as the tool intends to measure the 


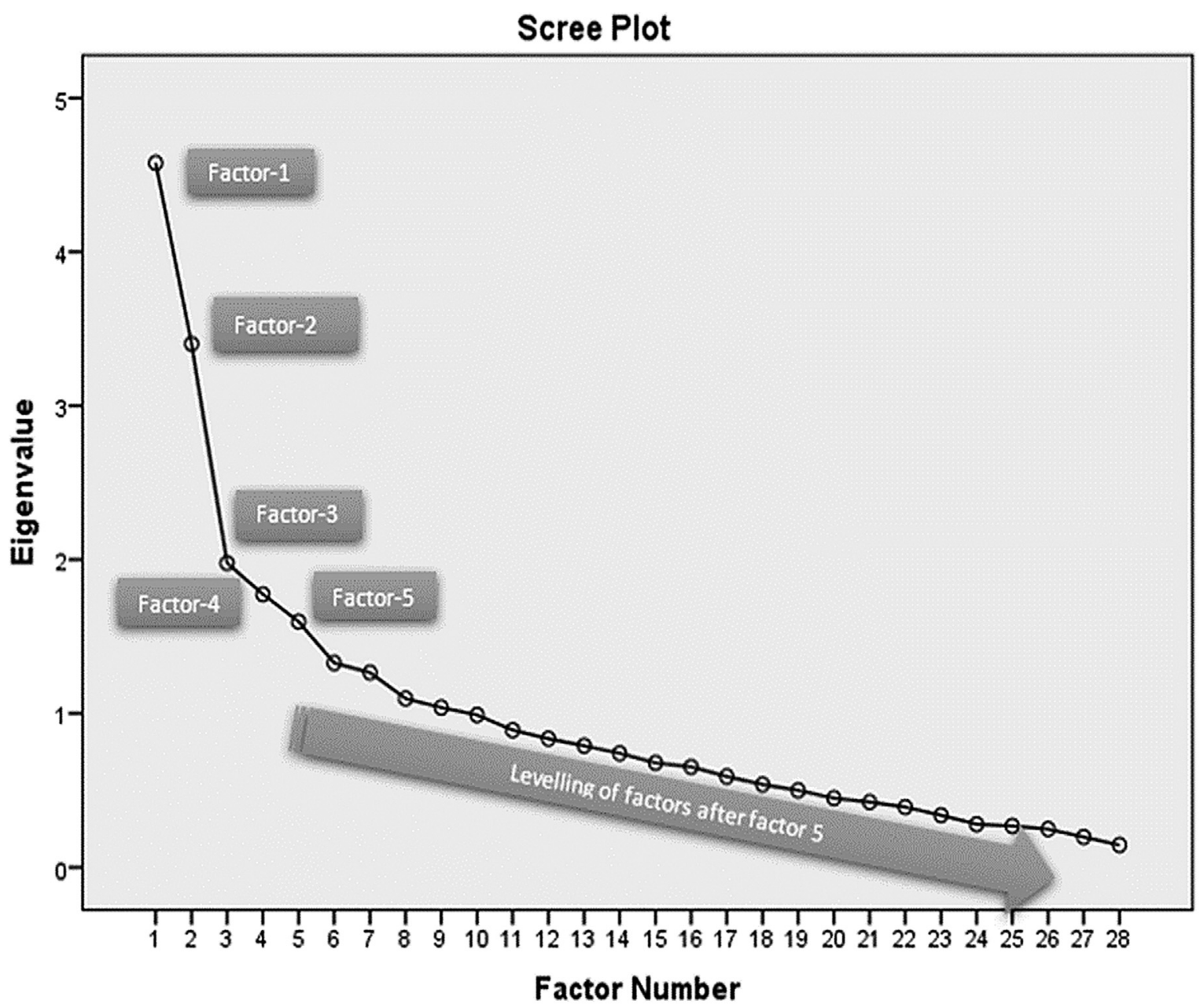

Fig. 3 Screen plot.

different aspects of distress associated with COVOD-19. Awareness of getting infected from a particular source of infection; fear and anxiety associated with test results; the uninvited stigma associated with COVID-19 infection (unlike other infections where public disclosure is not mandatory); the process of isolation and associated loss of health; and ability to maintain social reciprocity during this process and cope up with the stress of infection were some of the key concerns captured by the COVID Perception INterview Guide (COPING) tool. Moreover, this tool apart from giving measures of psychological status is also found to be a gentle and stepwise guide for interviewing patients infected with COVID-19. Hence, it also stands true for its name-COPING.

Unlike using only one approach for the computation of content validity, the present article gives a more holistic picture by calculating CVR, I-CVI, and S-CVI/UA from the responses of eight subject experts. ${ }^{37}$ Ratings of experts for ascertaining the items representing the domain were done using a CVR threshold of 0.75 in Lawshe Table. ${ }^{38}$ Studies have reported that the I-CVI of above 0.78 and S-CVI/UA of above 0.8 $(80 \%)$ indicate a good content validity. ${ }^{39}$ We eliminated the items with I-CVI below 0.70 and considered items with I-CVI above 0.78 . However, few items were revised based on experts' opinions with I-CVI between 0.70 and 0.78 . S-CVI/UA was satisfactory with a value above 0.8 . Following this, the factor analysis was conducted which gives fair and reasonable criteria for the selection of items with optimal performance. We found that eight items should be deleted from the tool. Thus, the credible underlying constructs for the set of items were recognized using factor analysis. With the deletion of five more items, Cronbach's $\alpha$ of above 0.7 was achieved. Our study supported that the items can be befittingly divided into five categories which were awareness, grief/bereavement, stigma, social reciprocity, and stress adaptation/coping. The satisfactory internal consistency (Cronbach's $\alpha$ value of 0.702 ) further reinforced that the reliability of the tool was good.

\section{Limitation}

An online self-administration by subjects creates recall bias, response bias, and under-/overratings. Online reporting has limited generalizability as it is limited to subjects familiar with handling smartphones or laptops. However, considering the nature of illness and the stringent COVID-19 protocols in place, the online self-administration may be justified. Also, the application of the COPING tool is valid in 
a clinical setting and may not be generalized to other settings. The items of the scale may appear vague but have validity in terms of acute settings where grief is a substantial component and could help is overall interview process by providing a channel for ventilation. The findings are from a single center and there is a possibility of overfactoring due to certain items having eigenvalues more than unity, and the limited variability as being explained by the scale. Further, perception of COVID-19 can be assessed from anthropological, sociological, and community mental health perspectives which were not touched upon in the current tool. Certain items may not be measuring the mental health impact but these were prominently striking during the qualitative interviews. This tool is not intended to measure mental health disorders such as depression, anxiety, etc., but focuses on the acute and subtle psychological domains. However, for researchers interested in measuring both the dimensions, we recommend the supplementation of established tools for screening mental health disorders along with COPING tools such as versions of the Primary Health Questionnaire.

\section{Conclusion}

COPING is a unique, easy-to-use, and simple self-administered tool which can be utilized by health care professionals to understand the perception of COVID-19 among acutely infected individuals as well as assist the health care workers to interview them. The tool has a high content validity and satisfactory construct validity with good reliability. The factor analysis strengthened the tool by recategorizing items in proper domains and deleting superfluous items. Additionally, this tool being tested among individuals acutely diagnosed with COVID-19 delivers an advantage of applying among other COVID-19-like illnesses as well to understand the psychological perception of similar infection. Further, the tool is validated in Hindi language, therefore appropriate language translation is recommended for its use in different geographical settings.

\section{Note}

For using the COPING tool in Hindi language, please contact the corresponding author Dr. Roshan Sutar.

\section{Authors' Contributions}

R.S. and G.S. are primarily responsible for designing and data collection of the study. R.S. and A.L. have contributed to the data analysis, manuscript writing, and proof reading. G.S., S.C., R.S., and A.L. have equally contributed for the research.

\section{Funding}

None.

\section{Conflict of Interest}

None declared.

\section{Acknowledgments}

We thank all the subjects who consented to this research and all the staff members who helped in coordinating the study. We also acknowledge and thank to Dr. Abhijit Pakhre, Dr. Vaibhav Ingle, Dr. Pooja Chaudhary, Dr. Parneet Kaur, and Mr. Neelesh Shrivastava for their contribution to data acquisition from few study participants.

\section{References}

1 Hiscott J, Alexandridi M, Muscolini M, et al. The global impact of the coronavirus pandemic. Cytokine Growth Factor Rev 2020; 53:1-9

2 Chakraborty I, Maity P. COVID-19 outbreak: migration, effects on society, global environment and prevention. Sci Total Environ 2020;728:138882

3 Srivastava N, Baxi P, Ratho RK, et al. Global trends in epidemiology of coronavirus disease 2019 (COVID-19). Coronavirus Dis 2019 COVID-19 2020:9-21

4 Sarwar A, Imran M. Prioritizing infection prevention and control activities for SARS-CoV-2 (COVID-19): a multi-criteria decisionanalysis method. J Healthc Leadersh 2021;13:77-84

5 Mackenzie JS, Smith DW. COVID-19: a novel zoonotic disease caused by a coronavirus from China: what we know and what we don't. Microbiol Aust 2020:MA20013

6 Tabish SA. COVID-19 pandemic: emerging perspectives and future trends. J Public Health Res 2020;9(01):1786

7 World Health Organization. Accessed August 12, 2021 at: https:// covid19.who.int/region/searo/country/in

8 MoHFW| Home. Accessed April 2, 2021 at: https://www.mohfw. gov.in/

9 Ghosh A, Nundy S, Mallick TK. How India is dealing with COVID-19 pandemic. Sens Int 2020;1:100021

10 Varghese GM, John R. COVID-19 in India: moving from containment to mitigation. Indian J Med Res 2020;151(2 \& 3):136-139

11 Siddiqui AF, Wiederkehr M, Rozanova L, et al. Situation of India in the COVID-19 pandemic: India's initial pandemic experience. Int J Environ Res Public Health 2020;17(23):8994

12 Shah K, Kamrai D, Mekala H, et al. Focus on mental health during the coronavirus (COVID-19) pandemic: applying learnings from the past outbreaks. Cureus 2020;12(03):e7405

13 Sahoo S, Mehra A, Dua D, et al. Psychological experience of patients admitted with SARS-CoV-2 infection. Asian J Psychiatr 2020;54:102355

14 Dubey S, Biswas P, Ghosh R, et al. Psychosocial impact of COVID-19. Diabetes Metab Syndr 2020;14(05):779-788

15 Wilson W, Raj JP, Rao S, et al. Prevalence and predictors of stress, anxiety, and depression among healthcare workers managing COVID-19 pandemic in India: a nationwide observational study. Indian J Psychol Med 2020;42(04):353-358

16 Rajkumar RP. COVID-19 and mental health: a review of the existing literature. Asian J Psychiatr 2020;52:102066

17 Sharma S, Kundu A, Basu S, Shetti NP, Aminabhavi TM. Indians vs.COVID-19: the scenario of mental health. Sens Int 2020; $1: 100038$

18 Grover S, Sahoo S, Mehra A, et al. Psychological impact of COVID19 lockdown: an online survey from India. Indian J Psychiatry 2020;62(04):354-362

19 Dsouza DD, Quadros S, Hyderabadwala ZJ, Mamun MA. Aggregated COVID-19 suicide incidences in India: fear of COVID-19 infection is the prominent causative factor. Psychiatry Res 2020;290:113145

20 Madurai Elavarasan R, Pugazhendhi R. Restructured society and environment: a review on potential technological strategies to control the COVID-19 pandemic. Sci Total Environ 2020;725:138858 
21 Cheval S, Mihai Adamescu C, Georgiadis T, et al. Observed and Potential Impacts of the COVID-19 Pandemic on the Environment. Int J Environ Res Public Health 2020;17(11):4140

22 Dutta A, Fischer HW. The local governance of COVID-19: disease prevention and social security in rural India. World Dev 2021; 138:105234

23 Kar SK, Yasir Arafat SM, Kabir R, et al. Coping with mental health challenges during COVID-19. In: Saxena SK, ed. Coronavirus Disease 2019 (COVID-19): Epidemiology, Pathogenesis, Diagnosis, and Therapeutics. Singapore: Springer; 2020:199-213

24 Kumaravel SK, Subramani RK, Jayaraj Sivakumar TK, et al. Investigation on the impacts of COVID-19 quarantine on society and environment: Preventive measures and supportive technologies. 3 Biotech 2020;10:393

25 Dalal PK, Roy D, Choudhary P, Kar SK, Tripathi A. Emerging mental health issues during the COVID-19 pandemic: An Indian perspective. Indian J Psychiatry 2020;62(Suppl 3):S354-S364

26 Pedrosa AL, Bitencourt L, Fróes ACF, et al. Emotional, Behavioral, and Psychological Impact of the COVID-19 Pandemic. Front Psychol 2020;11:566212

27 Gupta L, Gasparyan AY, Misra DP, Agarwal V, Zimba O, Yessirkepov M. Information and Misinformation on COVID-19: a cross-sectional survey study. J Korean Med Sci 2020;35(27):e256

28 Anwar A, Malik M, Raees V, Anwar A. Role of mass media and public health communications in the COVID-19 pandemic. Cureus 2020;12(09):e10453

29 Gupta S, Sahoo S. Pandemic and mental health of the front-line healthcare workers: a review and implications in the Indian context amidst COVID-19. Gen Psychiatr 2020;33(05):e100284

30 Krishnamoorthy Y, Nagarajan R, Saya GK, Menon V. Prevalence of psychological morbidities among general population, healthcare workers and COVID-19 patients amidst the COVID-19 pandemic: a systematic review and meta-analysis. Psychiatry Res 2020;293:113382
31 He Q, Fan B, Xie B, et al. Mental health conditions among the general population, healthcare workers and quarantined population during the coronavirus disease 2019 (COVID-19) pandemic. Psychol Health Med 2020;27(01):186-198

32 Rossi R, Socci V, Pacitti F, et al. Mental health outcomes among healthcare workers and the general population during the COVID19 in Italy. Front Psychol 2020;11;608986

33 Vahedian-Azimi A, Moayed MS, Rahimibashar F, Shojaei S, Ashtari S, Pourhoseingholi MA. Comparison of the severity of psychological distress among four groups of an Iranian population regarding COVID-19 pandemic. BMC Psychiatry 2020;20 (01):402

34 Zandifar A, Badrfam R, Yazdani S, et al. Prevalence and severity of depression, anxiety, stress and perceived stress in hospitalized patients with COVID-19. J Diabetes Metab Disord 2020;19(02): $1-8$

35 Li J, Li X, Jiang J, et al. The effect of cognitive behavioral therapy on depression, anxiety, and stress in patients with COVID-19: a randomized controlled trial. Front Psychiatry 2020;11:580827

36 Taquet M, Luciano S, Geddes JR, Harrison PJ. Bidirectional associations between COVID-19 and psychiatric disorder: retrospective cohort studies of 62354 COVID-19 cases in the USA. Lancet Psychiatry 2021;8(02):130-140

37 Almanasreh E, Moles R, Chen TF. Evaluation of methods used for estimating content validity. Res Social Adm Pharm 2019;15(02): 214-221

38 Ayre C, Scally AJ. Critical values for Lawshe's content validity ratio: revisiting the original methods of calculation. Meas Eval Couns Dev 2014;47:79-86

39 Polit DF, Beck CT, Owen SV. Is the CVI an acceptable indicator of content validity? Appraisal and recommendations. Res Nurs Health 2007;30(04):459-467 